\title{
ABSTRACT COMPUTABILITY AND ITS RELATION TO THE GENERAL PURPOSE ANALOG COMPUTER (SOME CONNECTIONS BETWEEN LOGIC, DIFFERENTIAL EQUATIONS AND ANALOG COMPUTERS)
}

\author{
BY \\ MARIAN BOYKAN POUR-EL(1)
}

\begin{abstract}
Our aim is to study computability from the viewpoint of the analog computer. We present a mathematical definition of an analog generable function of a real variable. This definition is formulated in terms of a simultaneous set of nonlinear differential equations possessing a "domain of generation." (The latter concept is explained in the text.) Our definition includes functions generated by existing general-purpose analog computers. Using it we prove two theorems which provide a characterization of analog generable functions in terms of solutions of algebraic differential polynomials. The characterization has two consequences. First we show that there are entire functions which are computable (in the sense of recursive analysis) but which cannot be generated by any analog computer in any interval-e.g. $1 / \Gamma(x)$ and $\sum_{n=1}^{\infty}\left(x^{n} / n^{\left(n^{3}\right)}\right)$. Second we note that the class of analog generable functions is very large: it includes special functions which arise as solutions to algebraic differential polynomials. Although not all computable functions are analog generable, a kind of converse holds. For entire functions, $f(x)=\sum_{i=0}^{\infty} b_{i} x^{i}$, the theorem takes the following form. If $f(x)$ is analog generable on some closed, bounded interval then there is a finite number of $b_{k}$ such that, on every closed bounded interval, $f(x)$ is computable relative to these $b_{k}$. A somewhat similar theorem holds if $f$ is not entire.

Although the results are stated and proved for functions of a real variable, they hold with minor modifications for functions of a complex variable.
\end{abstract}

Introduction. This work represents a chapter in the development of a mathematical theory of the analog computer. As stated in the abstract, the definition of analog generable function which we present is expressed in terms of a simultaneous set of nonlinear differential equations. We will see that it includes functions generated by existing general purpose analog computers-i.e., the electronic analog computer and the mechanical differential analyzer. Our definition with its "domain of generation" appears to differ considerably from the approach taken in [17]. We have found our approach necessary for reasons stated in footnotes 4 and 12.

Received by the editors April 12, 1971 and, in revised form, June 20, 1972. 34Al0.

AMS (MOS) subject classifications (1970). Primary 02F50, 02F99; Secondary 26A42, 33A15,

Key words and phrases. General purpose analog computer, recursive function, recursive analysis, computable function of a real variable, algebraic differential polynomial, Cauchy-Peano existence theorem for differential equations, Picard-Lindelöf theorem, transcendental field extensions, Gamma function.

(1) Supported in part by NSF GP 8829. The results were presented at a joint seminar on complex variables, differential equations and mathematical logic (Fall 1970) at the University of Minnesota. An abstract appears in the Notices of the American Mathematical Society, February 1970. 
One of our main concerns is to relate analog generable functions to abstract computability. Here one has to be a bit careful. In an analog computer the variables vary continuously. However, the basic concept of abstract computability is formulated in terms of computable functions from nonnegative integers to nonnegative integers. In order to relate these two concepts the following remarks may be helpful.

As is well known, the concept of abstract computability represents the distillation of several equivalent formulations-due to Turing [22], HerbrandGödel [10], Church, Markov [13] and others. Interestingly enough no serious attempt seems to have been made to formulate a notion of computability based on analog computers. This is certainly, not because the analog computer was unknown at that time, for the results of William Thomson, Lord Kelvin [20] date back to 1876 and the work of Vannevar Bush [2] goes back to 1931.(1a) By co trast, the digital computer fared much better than the analog machine. We recall that on Turing's approach, computability means computability by a most general type of digital computer-the Turing Machine. The equivalence of the above-mentioned formulations of Turing, Herbrand-Gödel, etc. provides us with our definition of "computable function from nonnegative integers to nonnegative integers". This definition leads naturally to a definition of "computable real number" and "computable function of a real variable". For example, to obtain "computable real" one merely "effectivizes" the classical constructions obtained from the foundations of mathematics-Cauchy sequence, Dedekind cut, etc. [16]. Similarly by effective approximation techniques one arrives at a definition of a computable function of a real variable (cf. [5]). Thus we have introduced recursive analysis.

Our concept of analog generability will be related to abstract computability via "computable function of a real variable". For technical convenience we do not use the definitions given in the literature. Rather we formulate our own definitions-Definitions 3 and 6-below. We use our definitions to give easy proofs that certain functions are computable. Computability on our definition is equivalent to computability relative to the more usual definitions [5]-Pour-El, Caldwell (to appear).

In our opinion interest in the topic of this paper stems from the following three considerations. The first is conceptual. As indicated above this research may be regarded as a first step in a study of an alternative approach to the concept of computability - an approach motivated by existing analog computers. Thus eventually we hope to do for existing analog computers what Turing did for digital computers. But much more is involved. For as we know the distinguishing feature of an analog computer-as opposed to a digital computer-is that the variables change continuously. Thus on this approach we can expect computable function of a real variable to be the fundamental concept explicated: computable

(1a) Shannon [17] is not concerned with abstract computability. 
functions from nonnegative integers to nonnegative integers will be a derived concept. This is, of course, a reversal of the procedure employed in connection with existing formulations of abstract computability. For as we have seen, existing formulations of computability follow along the traditional foundational development of mathematics-from 0 and the successor through functions from $N$ to $N$ to a definition of real number and a function of a real variable.(2) They proceed by effectivizing the corresponding classical constructions. The consequences of the aforementioned reversal-for the foundations of mathematics and for research in higher order computability as studied by logicians-appear to be of considerable interest. We do not wish to give the impression that we are against the traditional approach in any of its formulations. This approach is exceedingly fruitful. Furthermore it satisfies man's desire for simplicity and order. Our point is merely this. It may be possible to conceive that nature generates a computable function of a real variable directly and not necessarily by approximation as in the traditional approach. We believe it is of some interest to investigate this phenomenon.

The second consideration is technical: we believe that the techniques employed here may be of some independent interest. We have found it useful to combine the methods and results of classical analysis and differential equations with those of recursive function theory.(3) Work by Pólya, Hölder, Cauchy-Peano, Weierstrass, and others find application here. In some cases-e.g. in the use of Pólya's theorem and the Cauchy-Peano existence theorem for differential equations-the original result is used. In other cases, an effective-i.e. recursion-theoreticversion of the result is employed. In addition some algebraic results are also evident in our work. However this is not unusual in logical investigations.

Third, and most obvious, is the following consideration. We believe that it is of some interest to users of analog computers to investigate, from the point of view of abstract computability, the properties of the functions their devices generate. We further believe that this will also be of interest to users of digital and hybrid machines. For recently experts in digital computers have become increasingly concerned with hybrids-i.e. computers combining the features of both analog and digital machines-in an effort to maximize the advantages of both types of devices.

Survey of results. Throughout this paper we assume that our functions are continuous, with continuous derivatives. However it is not necessary to make such stringent requirements. We realize that some logicians may be unfamiliar with differential equations and conversely. For this reason, painstaking-and even tedious-attention is paid to detail in many sections of this work.

The plan of the paper is as follows. In $\$ 1$ we give recursion-theoretic, and other definitions which will be used later in this work. The main novelty occurs in

(2) $N$ is the set of nonnegative integers.

(3) I.e., the theory of abstract computability 
Definition 3 and Definition 6. Here we give the definition of "computable function of a real variable"-from the point of view of abstract computability theory-which we remarked upon above. The section concludes with definitions concerned with algebraic differential polynomials and other well-known concepts.

In $\$ 2$ we give the basic definition of a function generated by a general purpose analog computer (Definition 10). We show that our definition encompasses in a natural way all functions which can be generated by existing general purpose analog computers-e.g. the electronic differential analyzer and the mechanical differential analyzer.

$\S \S 3$ and 4 contain the principal theorems of this paper. $\left({ }^{4}\right)$ Since an analog computer generates a function in a closed bounded interval, the following notation will be useful. Let $I$ and $J$ be closed bounded intervals of the real line with nonempty interiors.

One of the main results of $\$ 3$ is the following: there are entire computable functions which cannot be generated by any general purpose analog computer on any $I$ however small. As examples we cite $1 / \Gamma(x)$ and $\sum_{n=1}^{\infty} x^{n} / n^{\left(n^{3}\right)}$. This result is a consequence of the following local characterization of analog generable functions in terms of algebraic differential polynomials.

(1) If $f$ is analog generable on $I$, then there is a $J \subseteq I$ such that, on $J, f$ satisfies an algebraic differential polynomial.

(2) If for some $I, f$ satisfies an algebraic differential polynomial on $I$, then there is a $J \subseteq I$ such that $f$ is analog generable on $J$.

The consequence is obtained by applying a theorem of Hölder and/or Pólya to (1) above. On the other hand (2) shows that the class of analog generable functions is very large. In fact many well-known special functions which arise as solutions to algebraic differential polynomials-e.g. Bessel functions-can be generated by a general purpose analog computer on every $I$ for which they are defined. This takes us far beyond the elementary functions $-e^{x}$, $\sin x$, etc.which are, of course, included.

The proofs of (1) and (2) use some results from differential equations together with some simple facts on the degree of transcendence of transcendental field extensions.

It is natural to ask whether every analog generable function is "essentially computable". We answer this question in the affirmative in $\$ 4$. (See Theorems 7

(4) There is apparently no counterpart to $\$ 4$ in Shannon [17]. Some statements similar to (1) and (2) above appear to be proved by Shannon [17] on the basis of his Theorem I. Unfortunately there is a gap in his proof of (1). For a detailed account of the gap see footnote 12. To fix this gap it was not only necessary to change Shannon's proof but to introduce the previously mentioned definition which includes "domain of generation". In \$2 we show, not only that existing analog computers possess this concept but that they would be useless without it. As indicated above different mathematical concepts than in [17] are used in the proofs. 
and 8.) One interesting sidelight of the work in $\$ 4$ is that it provides criteria which allow us to conclude immediately that many well-known functions are, in fact, computable in the sense of recursive analysis. For entire functions $f(x)$ $=\sum_{i=0}^{\infty} a_{i} x^{i}$ the criterion is quite simple. If each $a_{i}$ is a computable real and if $f(x)$ satisfies an algebraic differential polynomial then $f(x)$ is computable on every closed bounded interval. Actually, as we will see in $\$ 4$, the results can be extended to functions which are not necessarily entire.

Although the results of this paper are given for functions of a real variable, they hold-with trivial modifications-for functions of a complex variable.(5)

The paper concludes with a few remarks concerning further areas of research.

1. Preliminaries. In order to make this work self-contained and accessible to the nonspecialist, we summarize recursion-theoretic and other facts which will be used here. We do not presuppose extensive knowledge of recursion theory. We merely assume that the reader is acquainted with the class of recursive functions and its relation to the intuitive concept of "effectively calculable".

As explained in the introduction, our definition of "computable function of a real variable" differs from the usual ones-e.g. [5]. We first define computability on a closed bounded interval (Definition 3). Then we extend our definition to cover computability over the whole real line (Definition 6). A function is said to be computable if it is computable over the whole real line.

The definition which we give here is motivated by Weierstrass's well-known approximation theorem. In order to "effectivize" this theorem it is convenient to state it in a rather awkward form: if $\varphi$ is continuous on $[a, b]$, then there exists a sequence of polynomials $\left\{P_{n}(x)\right\}$ and an $N(M)$ such that, for all $n>N(M)$, $\left|\varphi(x)-P_{n}(x)\right|<1 / 2^{M}$. Definition 3 (below) represents an "effectivization" of this form of Weierstrass's theorem. It effectivizes both the sequence of polynomials (Definition 1) and the modulus of continuity (Definition 2).

Definition 1. A sequence of polynomials $\left\{P_{n}(x)\right\}$ is said to be recursively enumerable if there exist recursive functions $q, r, s$, and $d$ such that $(6)$

$$
P_{n}(x)=\sum_{j=0}^{d(n)}(-1)^{(s(n))_{j}} \frac{(q(n))_{j}}{1+(r(n))_{j}} x^{j}
$$

Definition 2. Let $\varphi$ be a continuous function on $[a, b]$. A sequence of polynomials $\left\{P_{n}(x)\right\}$ is said to converge recursively to $\varphi$ on $[a, b]$ if there exists a

(5) One note of caution. Do not use a Weierstress approximation by polynomials of a complex variable to define "computable function of a complex variable" as in Definitions 3 and 6. Weierstrass's theorem does not hold! Instead, require that the real and imaginary parts be computable. Here an obvious modification allows us to pass from functions of one real variable to functions of two real variables.

(6) $(a)_{j}$ is Kleene's notation: $(a)_{j}$ is the power to which the $j$ th prime $p_{j}$ appears in the prime factorization of $a$. (The number 2 is referred to as the 0 th prime.) $(0)_{j}=0$ for all $j$. 
recursive function $g$ such that, whenever $a \leq x \leq b$,

$$
\left|\varphi(x)-P_{n}(x)\right|<\frac{1}{2^{M}}
$$

for all $n \geq g(M)$.

Definition 3. Let $\varphi$ be continuous on $[a, b]$. Then $\varphi$ is computable on $[a, b]$ if there exists a sequence $\left\{P_{n}(x)\right\}$ of polynomials which is recursively enumerable and which converges recursively to $\varphi$ on $[a, b]$.

We now turn to defining the computability of $\varphi$ on the entire line. Note that it is not sufficient that $\varphi$ be computable on every closed bounded interval. We must require that $\varphi$ be uniformly computable. Thus by analogy to the above we are led to the following definitions.

Definition 4. A double sequence of polynomials $\left\{P_{N, n}(x)\right\}$ is said to be recursively enumerable if there exist recursive functions $q, r, s$, and $d$ such that

$$
P_{N, n}(x)=\sum_{j=0}^{d(N, n)}(-1)^{(s(N, n))_{j}} \frac{(q(N, n))_{j}}{1+(r(N, n))_{j}} x^{j}
$$

Definition 5. Let $\varphi$ be continuous on the real line. A double sequence of polynomials $\left\{P_{N, n}(x)\right\}$ is said to converge recursively to $\varphi$ if there exists a recursive function $g$ so that, whenever $-N \leq x \leq N$,

$$
\left|\varphi(x)-P_{N, n}(x)\right|<\frac{1}{2^{M}}
$$

if $n \geq g(N, M)$.

Definition 6. Let $\varphi$ be continuous on the real line. $\varphi$ is computable if there exists a recursively enumerable sequence of polynomials $\left\{P_{N, n}(x)\right\}$ which converges recursively to $\varphi$.

We now turn our attention from computability to "essential computability". In the next definition we assume that $u$ is a real number which can be written in decimal notation as $\pm(u(0) \cdot u(1) u(2) \cdots u(n) \cdots)$. We exclude the case in which the decimal expansion has the form $\pm(u(0) \cdot u(1) u(2) \cdots u(n) 9999 \cdots)$. Furthermore ' + ' or ' -' is employed according as $u \geq 0$ or $u<0$. Consider the sequence of partial sums

$$
\pm u(0), \pm \frac{10 u(0)+u(1)}{10}, \ldots, \pm \frac{10^{n} u(0)+10^{n-1} u(1)+\cdots+u(n)}{10^{n}} \cdots .
$$

Define the function $v$ by

$$
v(n)=10^{n}(u(0))+10^{n-1} u(1)+\cdots+u(n) .
$$

The function $v$ defined above is said to be the associate of the real number $u$.

It is now an easy matter to define essential computability in $u_{1}, \ldots, u_{k}$. One merely relativizes Definitions $1-6$ in the obvious way. For example:

A sequence of polynomials $\left\{P_{n}(x)\right\}$ is said to be essentially recursively enumera- 
ble in a finite set of reals $u_{1}, \ldots, u_{k}$ if there exist functions $q, r, s$ and $d$, each of which is recursive in the associates of $u_{1}, \ldots, u_{k}$ such that

$$
P_{n}(x)=\sum_{j=0}^{d(n)}(-1)^{(s(n))_{j}} \frac{(q(n))_{j}}{1+(r(n))_{j}} x^{j}
$$

The relativization of Definition 3 gives essential computability in $u_{1}, \ldots, u_{k}$ on $[a, b]$. The relativization of Definition 6 gives essential computability in $u_{1}, \ldots, u_{k}$ (over the whole real line). We leave it to the reader to make the appropriate modifications.

Our preliminary discussion of recursion theory closes with the following definition.

Definition 7. The sequence $a_{0}, a_{1}, \ldots, a_{n}, \ldots$ of reals is essentially computable in a finite set of reals $u_{1}, \ldots, u_{k}$ if there exist functions $q, r, s$ and $g$ each of which is recursive in the associates of $u_{1}, \ldots, u_{k}$ such that

$$
\left|a_{n}-(-1)^{s(n, m)} \frac{q(n, m)}{1+r(n, m)}\right|<\frac{1}{2^{k}} \text { for } m \geq g(n, k) .
$$

We recall the following definitions concerning algebraic differential polynomials.

Definition 8. An algebraic differential polynomial is an expression of the form

$$
\sum_{i=1}^{n} a_{i} x^{p_{i}} y^{q_{i}}\left(y^{\prime}\right)^{q_{11}} \cdots\left(y^{\left(k_{i}\right)}\right)^{q_{k_{i}}}
$$

where $a_{i}$ is a real number, $p_{i}, q_{0 i}, \ldots, q_{k_{i} i}$ are nonnegative integers and $y$ is a function of $x$.

Definition 9. A function $y(x)$ satisfies an algebraic differential polynomial if it satisfies an equation of the form $P\left(x, y, y^{\prime}, \ldots, y^{(n)}\right)=0$ where $P$ is an algebraic differential polynomial.

We conclude this section with the following well-known terminology. A function $f(x)$ is analytic on an interval $I$ if it is the restriction of a function of a complex variable which is analytic in a region including $I$; a function $f(x)$ is entire if it is the restriction of an entire function of a complex variable.

2. The basic definition. In this section we give our fundamental definition, $y(x)$ is generated by a general purpose analog computer (G.P.A.C.). The definition encompasses existing general purpose analog machines. We will see that it can be conceived of in terms of a set of simultaneous nonlinear differential equations. At first sight the relation between our definition and existing analog machines may seem a bit strange. In order to see that this is a natural generalization which includes existing G.P.A.C. we proceed as follows. First we give our definition of "function generated by a G.P.A.C." (Definition 10 below). Next we give a brief discussion of the nature of existing analog computers. This is followed by a discussion of a preliminary definition-which will be in terms of black boxes, 
feedback, etc. (It should be clear from this account that the existing G.P.A.C.'s are included under our preliminary definition.) We will see that we are led to a definition which is essentially graph-theoretic in character. Finally we relate our preliminary definition to the final definition which, as we have already remarked, involves nonlinear differential equations with a "domain of generation".

Notation. I, J: (with and without subscripts and superscripts) closed bounded intervals with nonempty interiors,

$$
\begin{aligned}
& c_{i j k}: \text { real numbers, } \\
& y_{0}: y_{0}(x) \equiv 1, \\
& y_{1}: y_{1}(x) \equiv x .
\end{aligned}
$$

(The last two items of notation are used in [17].)

Definition 10. The function $y(x)$ is generated by a G.P.A.C. on $I$ if there exists a set of functions $y_{2}(x), \ldots, y_{n}(x)$ and a set of initial conditions $y_{i}(a)=y_{i}^{*}$ where $a \in I$-such that:

(1) $\left\{y_{2}, \ldots, y_{n}\right\}$ is the unique solution on $I$ of a set of differential equations of the form

$$
A(x, \vec{y}) \frac{d \vec{y}}{d x}=b(x, \vec{y})
$$

satisfying the initial conditions.

(Here the vector $\vec{y}$ has components $y_{2}, \cdots, y_{n}, A(x, \vec{y})$ is an $(n-1) \times(n-1)$ matrix, and each entry of $A$ and $b$ is linear in $1, x, y_{2}, \cdots, y_{n}$ over the reals.)

(2) For some $i$ such that $2 \leq i \leq n, y(x) \equiv y_{i}(x)$ on $I$.

(3) $\left\langle a, y_{2}^{*}, \ldots, y_{n}^{*}\right\rangle$ has a domain of generation with respect to the equations of (1). See remark below.

Remark. The concept "domain of generation" will be explained at the end of this section-page 12-because we need some preliminaries to make it understandable. We will see that it is a very natural requirement to make.

We now turn our attention to existing analog computers. It is of ten said that a digital computer is any device in which the variables vary discretely whereas an analog computer is any device in which the variables vary continuously. Thus, on this rather vague definition, a drop of water issuing from a faucet at regular intervals may be conceived of as a digital computer. The literature on digital computers is more restrictive than that! Similarly-although an analog computer is often conceived of as any device in which the variables vary continuously-the literature on analog computers is much more restrictive. So what does the literature on analog computers conceive an analog computer to be? Clarence Johnson, in his well-known textbook on Analog Computer Techniques [9], divides the class of analog computers into two main categories. $(7)$ The first category consists of the special purpose machines. Here we find the planimeter, the wind

(7) This classification is employed by many authors. The reader is invited to consult the literature. 
tunnel and other special purpose machines. In this paper we will not be concerned with these specialized devices. Rather our attention will be focused on the second category, the class of general purpose analog computers. The main types of devices listed under the heading "general purpose analog computer" are the electronic differential analyzer and the mechanical (or electro-mechanical) differential analyzer. We recall that the electronic differential analyzer is also referred to as the "D.C. analog computer" or just as the "analog computer"-as this is probably the most well-known of the general purpose devices.

In order to understand the motivation behind Definition 10 it is useful to discuss briefly the structure of both the electronic differential analyzer and the mechanical differential analyzer. The electronic differential analyzer will be considered first; we will then make a few remarks to relate this discussion to the mechanical differential analyzer.

The functions generated by an electronic differential analyzer are functions of time: they are usually measured in terms of volts. This device is composed of a finite number of black boxes of the following types. The integrator is a one-input, one-output device with a setting for initial conditions: if we input $u(t)$ and set the initial condition $e(a)$ at time $t=a$, we will output $\int_{a}^{t} u(t) d t+e(a)$. There is also an adder, a two-input, one-output device: if $u(t)$ and $v(t)$ are the inputs then $u(t)+v(t)$ is the output. For each rational constant $k$, there is a constant multiplier. This is a one-input, one-output device: if we input $u(t)$ we obtain $k u(t)$ as output. Finally we have the variable multiplier, a two-input, one-output device: if we input $u(t)$ and $v(t)$ we obtain $u(t) \cdot v(t)$ as output.

The electronic differential analyzer is constructed from a finite number of the above-mentioned units. This is accomplished by interconnecting these units by wires or plugs. Feedback is, of course, permitted! Interconnections and their relation to feedback, the method of generating functions, as well as other matters, will be discussed in greater detail-in a more general setting-as part of our preliminary definition of a general purpose analog computer. Those interested in the hardware needed to construct an electronic differential analyzer are referred to standard texts [8], [9],-cf. [11], [18], [21].

The functions generated by a mechanical differential analyzer are also functions of time. Of course they are not measured in volts! Nevertheless, the mechanical differential analyzer possesses essentially the same basic units subject to the following modifications. First the hardware which realizes this analyzer is mechanical or electro-mechanical in nature, rather than electronic. (Since this paper is not concerned with hardware, this is not an essential difference for us.) Second the integrator is slightly more general. For a more detailed account of the structural features of a mechanical differential analyzer the reader is invited to consult [4].

We now turn our attention to a preliminary definition. This definition is motivated by the structure of the differential analyzers discussed above. Accord- 
ingly we will be concerned with the following units. $\left({ }^{8}\right)$ (Note that our functions are not necessarily functions of time.) We will consider functions of one variable only although our discussion is obviously applicable to functions of several variables.

1. Integrator. A two-input, one-output device with a setting for initial conditions. If $u(x)$ and $v(x)$ are inputs, we obtain as output $\int_{x_{0}}^{x} u(x) d v(x)+C$-where $C$ is a constant depending on the initial conditions and $\int_{x_{0}}^{x} u(x) d v(x)$ is the Riemann-Stieltjes integral.

2. Constant multiplier. For each real constant $k$, there is a device with one input and one output. If $u(x)$ is the input, then $k u(x)$ is the output.

3. Adder. A two-input, one-output device. If $u(x)$ and $v(x)$ are inputs, then $u(x)+v(x)$ is the output.

4. Variable multiplier. A two-input, one-output device. If $u(x)$ and $v(x)$ are inputs, then $u(x) \cdot v(x)$ is the output.

5. Constant function. A one-input, one-output device. If $u(x)$ is the input, then $C_{1}(x)$-where $C_{1}(x) \equiv 1$-is the output.

To construct a general purpose analog computer a finite number of these units must be interconnected. We require, as in practice, that two inputs and two outputs can never be interconnected. We say that $\mathfrak{A}$ is a general purpose analog computer if $\mathfrak{A}$ is a collection of $n-1$ units $U_{2}, \ldots, U_{n}\left({ }^{9}\right)$ which are interconnected so that each input is connected to at most one output. Thus the reader might envision a portion of the computer as follows. (Assume the input terminals are on the left and the output terminals are on the right.)

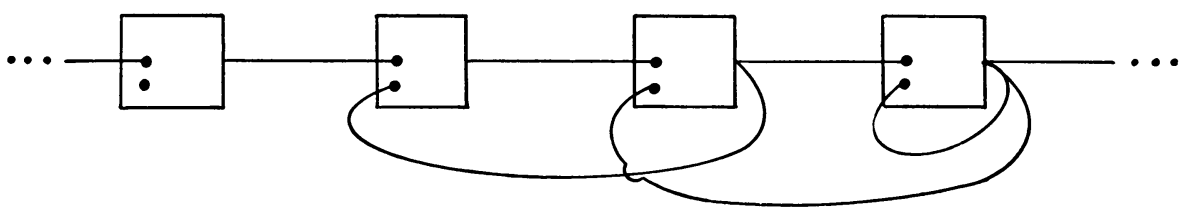

Note that feedback, which may be conceived of as a form of continuous recursion, is permitted! Without feedback this study would degenerate into an uninteresting academic exercise. Incidentally it is easy to describe feedback in terms of the units of an analog computer. We say that a general purpose analog computer $\mathfrak{A}$ with $n-1$ units-has feedback if it is not possible to enumerate the units of $\mathfrak{A}-$ viz. $U_{2}, \ldots, U_{n}$-in such a way that if the output of $U_{i}$ is connected to an input of $U_{j}$ then $i<j$. Denote the output of $U_{i}$ by $y_{i}$. We say that the function $u(x)$ is

(8) Contrary to engineering realities the units will be considered as idealized-and hence perfect-components.

(9) The reason for this rather peculiar notation is the following. It is convenient to refer to the output of $U_{i}$ as $y_{i}(x)$. But we have already established the following notation: $y_{0}(x) \equiv 1, y_{1}(x) \equiv x$. Thus we index our units beginning with 2 . 
generated by $\mathfrak{A}$ on $I(10)$ if we can prescribe initial conditions to the integrators of $\mathfrak{A}$ at $x=a$ for some $a \in I$ so that if $x$ is applied to every input not connected to an output, then for some $i,(2 \leq i \leq n)$, we have $y_{i}(x) \equiv u(x)$ on $I$. We say that $u(x)$ is generable by an analog computer on $I$ if there is some $\mathfrak{A}$ such that $u(x)$ is generated by $\mathfrak{A}$ on $I$.

Thus we conclude our preliminary sketch of "general purpose analog computer" and "function generated by a general purpose analog computer". At this point the reader will note that the definitions given here are essentially graphtheoretic in nature. We invite him to translate these concepts into rigorous graphtheoretic definitions for himself: it is a rather simple exercise.(11) For our purposes this translation will not be necessary as we will now prove Proposition 1 , a very simple proposition, which will partly explain the use of Definition 10 as the final form of our definition. Proposition 1 is related to [17, Theorem 1] which is concerned with Bush's mechanical differential analyzer. Before turning to the proposition, we wish to make a remark which will be useful in its proof.

Remark. The variable multiplier can be eliminated. This is because

$$
u(x) \cdot v(x)=\int_{a}^{x} u(x) d v(x)+\int_{a}^{x} v(x) d u(x)+u(a) v(a)
$$

Proposition 1. If a function $y(x)$ is generable on I in the "graph-theoretic" sense it is generable on $I$ in the sense of Definition 10.

Proof. We recall that in the previous remark we showed that the variable multiplier can be eliminated. Thus our analog computer has as units only: integrators, constant multipliers, the constant function $C_{1}$, and adders.

We first show that this holds if $y$ is the output of an integrator. Let $\mathfrak{A}$ be an analog computer with $n-1$ integrators having as outputs $y_{2}, \ldots, y_{n}$ respectively. Now each input of an integrator must be the output of one of the following: a constant multiplier, an adder, the constant function $C_{1}$, an integrator, or the independent variable. Thus the integrand of the $k$ th integrator is expressible as $\sum_{i=0}^{n} c_{i k}^{*} y_{i}$ for suitable constants $c_{i k}^{*}$. Similarly the variable of integration of the $k$ th integrator can be expressed as $\sum_{j=0}^{n} c_{j k}^{* *} y_{j}$. Thus

(10) An obvious modification of this can be made when dealing with functions of more than one variable. Simply require that precisely one of the independent variables be applied to each input not connected to an output.

(11) The reader versed in formal logic will note that the concepts sketched above can be "Gödelized" within the reals. For assume we have countably many integrators, countably many adders and countably many constant multipliers for each real constant. Then it is easy to define " $y$ is the $n$th integrator", " $y$ is the $n$th adder", " $y$ is the $n$th constant multiplier associated with the real $k$ ". Thus we can obtain " $y$ is a unit". From this it is easy to formulate " $y$ is an interconnection" and " $y$ is an analog computer". 


$$
\begin{aligned}
& y_{k}=\int_{a}^{x} \sum_{i=0}^{n} c_{i k}^{*} y_{i} d\left(\sum_{j=0}^{n} c_{j k}^{* *} y_{j}\right)+C, \\
& y_{k}=\int_{a}^{x} \sum_{i=0 ; j=0}^{n} c_{i k}^{*} c_{j k}^{* *} y_{i} d y_{j}+C .
\end{aligned}
$$

If we define $c_{i j k}$ so that $c_{i j k}=c_{i k}^{*} c_{j k}^{* *}$ then we have

$$
\frac{d y_{k}}{d x}=\sum_{i=0 ; j=0}^{n} c_{i j k} y_{i} \frac{d y_{j}}{d x}, \quad k=2, \ldots, n .
$$

It is easy to extend this result to cover the output of any unit. For suppose $y$ is the output of a unit of an analog computer $\mathfrak{A}$. Then, since $y(x)=\int_{a}^{x} 1 \cdot d y$ $+y(a), y$ can be considered as the output of an integrator in an analog computer $\mathfrak{A}^{\prime}$, obtained by adding the integrator associated with $\int 1 d y$ and suitably altering the connections.

We now discuss the concept domain of generation. Suppose that $\left\{y_{2}, \ldots, y_{n}\right\}$ is the unique solution on $I$ of a set of equations of the form

$$
A(x, \vec{y}) \frac{d \vec{y}}{d x}=b(x, \vec{y})
$$

satisfying the initial conditions $y_{i}(a)=y_{i}^{*}$. Then the use of analog computers in the real world suggests that each point $\left\langle x^{* *}, u_{2}^{* *}, \ldots u_{n}^{* *}\right\rangle$ sufficiently close to $\left\langle a, y_{2}^{*}, \ldots, y_{n}^{*}\right\rangle$ should provide initial conditions for a unique solution of $\left(\mathrm{E}_{0}\right)$ on some interval $I^{* *}$. This is because the initial conditions represent initial settings on the integrators. (In practice the operator is allowed to vary the initial conditions on an analog computer slightly.) More precisely we require the following. There are closed intervals $J_{1}, \ldots, J_{n}$-with nonempty interiors-such that $\left\langle a, y_{2}^{*}, \ldots, y_{n}^{*}\right\rangle$ is an interior point of $J_{1} \times J_{2} \times \cdots \times J_{n}$. Furthermore whenever $\left\langle x^{* *}, u_{2}^{* *}, \ldots, u_{n}^{* *}\right\rangle \in J_{1} \times J_{2} \times \cdots \times J_{n}$ there exists a set of functions $\left\{u_{2}, \ldots, u_{n}\right\}$ such that:

(i) $u_{i}\left(x^{* *}\right)=u_{i}^{* *}$ for $i=2, \ldots, n$.

(ii) $\left\{u_{2}, \ldots, u_{n}\right\}$ satisfies $\left(\mathrm{E}_{0}\right)$ on some $I^{* *}$ for which $x^{* *} \in I^{* *}$.

(iii) $\left\{u_{2}, \ldots, u_{n}\right\}$ is locally unique-i.e. unique on $I^{* *}$ and on any subinterval of $I^{* *}$ containing $x^{* *}$.

$J_{1} \times J_{2} \times \cdots J_{n}$ is called a domain of generation of $\left\langle a, y_{2}^{*}, \ldots, y_{n}^{*}\right\rangle$ with respect to $\left(\mathrm{E}_{0}\right)$ and is denoted by $D g$.

Remark. The reader versed in recursion-theoretic techniques will note with surprise that we have taken as units constant multipliers for every real constant $k$. Thus we will see that the functions generated by an analog computer become "essentially computable" as defined in $\S 1$ rather than computable. One reason for our choice of units is to emphasize the fact that even if we allow ourselves all real numbers there exist computable entire functions which cannot be generated by any G.P.A.C.-e.g. $1 / \Gamma(x)$. The more constructively oriented reader may wish to restrict the class of constant multipliers to a class of constants which can be more effectively defined. He will find that, modulo trivial and obvious modifications, our work will hold for his analog computers. 
Let $F$ be the class of functions differentiable on $[a, x]$. Recall that Definition 10 makes sense whenever $y_{2}, \ldots, y_{n}$ are members of $F$. However the proof of Proposition 1 makes use of the Riemann-Stieltjes integral. Thus, in order to prove it we must restrict our attention to a subclass $G$ of $F$ having the following properties. If $u \in G$ and $v \in G$ then

(a) $\int_{a}^{x} u(x) d v(x)$ exists;

(b) if $w(x)=\int_{a}^{x} u(x) d v(x)$, then $d w / d x=u(x) d v / d x$.

Certainly these assumptions hold if $G$ is the class of functions with continuous derivatives on $[a, x]$ (see [1, pp. 191-232]).

There are two approaches which one can take to the range of variables of Definition 10. The first approach is to restrict the use of Definition 10 to functions which are members of $G$. The second is to allow the $y$ 's of Definition 10 to range over all of $F$. However Definition 10 will be associated with existing hardware differential analyzers when the $y$ 's are members of $G$. For the purposes of this paper it does not matter which approach is employed.

It ought to be remarked that Definition 10 can be extended to cover the case in which $y_{2}, \ldots, y_{n}$ are functions of more than one variable in an obvious way. We leave the precise statement of the extended definition to the reader.

3. Entire functions which are computable but not analog generable. Our primary aim in this section is to give an example of an entire computable $f$ such that for every $I, f \uparrow I$ cannot be generated by a G.P.A.C. Many examples-inclưding $1 / \Gamma(x)$ - can be found in Theorem 3 below. The proof of Theorem 3 depends on Theorem 2 which relates generability by a G.P.A.C. to solutions of an algebraic differential polynomial. The section concludes with Theorem 4. Roughly Theorem 4 may be conceived of as a converse to Theorem 2 . As a consequence of these two theorems we become aware of the relation between analog generable functions and functions satisfying an algebraic differential polynomial. From Theorem 4 it follows that the class of analog generable functions is very comprehensive.

It ought to be remarked that the techniques in classical analysis and differential equations used to obtain Theorem 3 may be viewed as a replacement for the technique of diagonalization of recursion theory. Such a replacement appears to be necessary as it is difficult to conceive of using diagonalization itself to obtain an entire function.

Recall that $I$ and $J$-with and without subscripts and superscripts-are closed bounded intervals with nonempty interiors.

Theorem 2.(12) If $y$ is generable on I by an analog computer then there is an $I^{\prime} \subseteq I$ such that, on $I^{\prime}, y$ satisfies an algebraic differential polynomial.

(12) A statement somewhat similar to this appears as part of Theorem II [17, p.342]. We believe that there is a serious gap in the brief proof which appears on the top of p. 343. For this reason we have found it necessary to proceed along entirely different lines. Our work is considerably more involved than that sketched in [17]. As remarked in the text it uses matrix theory, the Cauchy-Peano 
Proof. By Definition 10 there is a $\left\{y_{2}, \ldots, y_{n}\right\}$ such that $\left\{y_{2}, \ldots, y_{n}\right\}$ satisfies a set of equations of the form

$$
A(x, \vec{y}) \frac{d \vec{y}}{d x}=\vec{B}(x, \vec{y})
$$

where $y=y_{2}, \vec{y}$ is the column vector with components $y_{2}, \ldots, y_{n} ; A$ is an $(n-1) \times(n-1)$ matrix whose components are linear in $1, x, y_{2}, \ldots, y_{n} ; \vec{B}$ is an $n-1$ dimensional vector whose components are linear in $1, x, y_{2}, \ldots, y_{n}$. We can consider the entries of $A$ and $\vec{B}$ as elements of the ring generated from the reals by adjoining $x, y_{2}, \ldots, y_{n}$.

Let $y_{2}(a)=y_{2}^{*}, \ldots, y_{n}(a)=y_{n}^{*}$ be the initial conditions guaranteed by Definition 10.

The proof is detailed. Hence it may be of use to give a brief summary. We first show that $\operatorname{det} A \not \equiv 0$ on $I$. This is proved by contradiction. Roughly we use the Cauchy-Peano existence theorem to show the following. If $0<\operatorname{rank} A<n-1$ then (E) would have more than one solution satisfying a certain set of initial conditions. Note that this set of initial conditions will, in general, differ from $y_{2}(a)=y_{2}^{*}, \ldots, y_{n}(a)=y_{n}^{*}$. We show only that considered as a point in $n$-space it lies in the domain of generation of (E) with respect to $\left\langle a, y_{2}^{*}, \ldots, y_{n}^{*}\right\rangle$. But by definition elements of the domain of generation lead to unique solutions of (E). Once we have demonstrated that $\operatorname{det} A \not \equiv 0$ our theorem follows from some simple facts on the degree of transcendence of transcendental field extensions.

Let $J_{1} \times \cdots \times J_{n}$ be a domain of generation of $\left\langle a, y_{2}^{*}, \ldots, y_{n}^{*}\right\rangle$ with respect to (E). Let $J_{1}^{0}$ be a subset of $J_{1}$ such that

(i) $a$ is an interior point of $J_{1}^{0}$;

existence theorem for differential equations, and some facts concerning the degree of transcendence of transcendental field extensions.

Let us make a few comments stating the reason we believe there is a gap. In the case of Sylvester's method one must rule out the following possibility: at some stage $s$, after the first $s$ variables have been eliminated the system of equations in the remaining $n^{2}-2 n-s$ variables vanishes identically. If these variables were not related to each other by differentiation then one could conclude that given any values for the remaining $n^{2}-2 n-s$ variables one could find values for the first $s$ variables so that the $(n-1)^{2}$ equations would be satisfied. Thus the equations would not have a unique solution, contradicting the basic assumption. However, and this is the main point, it is conceivable that the system of $(n-1)^{2}$ equations may have many solutions if we ignore the fact that the $n^{2}-2 n$ variables are related by differentiation, but only one solution if we consider that they are related by differentiation. This possibility ought to be ruled out. (For an account of the complications involved in Sylvester's method see van der Waerden's Modern Algebra (English translation) vol. II, $\$ \$ 77$ and 78 .) It ought to be remarked that other methods of elimination have their problems which prevent the proof from being completely trivial. For example, in using Cramer's rule we must prove that the determinant of the coefficient matrix is not identically zero.

In conclusion, this author believes that it is preferable not to use a technique which requires additional differentiation on the set of equations which demonstrate that $y_{2}, \ldots, y_{n}$ can be generated by a G.P.A.C., for such a procedure requires additional assumptions on the differentiability of the $y_{i}$, which is not necessary for the proof of the theorem. 
(ii) if $x \in J_{1}^{0}$ then $\left\langle x, y_{2}(x), \ldots, y_{n}(x)\right\rangle \in J_{1} \times \cdots \times J_{n}$.

Let $I_{1}=J_{1}^{0} \cap I$.

We first show that $\operatorname{det} A \not \equiv 0$ on $I_{1}$. For suppose not:

Case 1. Suppose $A$ is of rank 0 on $I_{1}$. Then each entry of $A$ is zero on $I_{1}$. Thus we have

$$
\begin{aligned}
& 0=b_{1}\left(x, y_{2}(x), \ldots, y_{n}(x)\right) \\
& 0=b_{2}\left(x, y_{2}(x), \ldots, y_{n}(x)\right) \\
& \vdots \\
& 0=b_{n-1}\left(x, y_{2}(x), \ldots, y_{n}(x)\right)
\end{aligned}
$$

on $I_{1}$. Hence $b_{1}, \ldots, b_{n-1}$ are identically zero on $I_{1}$. Now $y_{2}(x), \ldots, y_{n}(x)$ cannot be the unique solution of a set of equations of the form $0=0$ no matter what the initial conditions may be. So this case cannot hold.

Case 2. Suppose $A$ is of rank $r$ where $0<r<n-1$.

By relabelling we can assume that $\operatorname{det} B$, where

$$
B=\left\|\begin{array}{ccc}
a_{11} & \cdots & \\
\vdots & & \\
& & a_{r r}
\end{array}\right\|,
$$

is not identically zero on $I_{1}$. Let $\left\langle x_{0}, y_{2}\left(x_{0}\right), \ldots, y_{n}\left(x_{0}\right)\right\rangle$ be such that $\operatorname{det} B$ evaluated at $\left\langle x_{0}, y_{2}\left(x_{0}\right), \ldots, y_{n}\left(x_{0}\right)\right\rangle$ is not zero. Then by continuity there is an $I_{2} \subseteq I_{1}$ so that

(iii) $x_{0} \in I_{2}$;

(iv) det $B\left(x, y_{2}(x), \ldots, y_{n}(x)\right) \neq 0$ for any $x \in I_{2}$.

We can assume without loss of generality that $x_{0}$ is an interior point of $I_{2}$. We now restrict our attention to the interval $I_{2}$. By a well-known fact concerning matrices with entries which are in a commutative ring, for each $l>r$ and for all $k$ we can find $f_{l r+1}, f_{l l}, \ldots, f_{l k}$ such that $f_{l r+1} \neq 0$ and

$$
f_{l r+1} a_{l k}=f_{l 1} a_{1 k}+\cdots+f_{l r} a_{r k} .
$$

Note that in the above application $f_{l r+1}=\operatorname{det} B$. Hence on $I_{2}$, for all $k$, if $l>r$,

$$
a_{l k}=\left(f_{l 1} / f_{l r+1}\right) a_{1 k}+\cdots+\left(f_{l r} / f_{l r+1}\right) a_{r k},
$$

i.e. if $l>r$, the lth row is a linear combination of the first $r$ rows. Thus on $I_{2}$ our set of equations reduces to

$$
\begin{aligned}
a_{11} d y_{2} / d x+\cdots+a_{1 n-1} d y_{n} / d x & =b_{1}\left(x, y_{2}(x), \ldots, y_{n}(x)\right) \\
\vdots & \\
a_{r 1} d y_{2} / d x+\cdots+a_{r n-1} d y_{n} / d x & =b_{r}\left(x, y_{2}(x), \ldots, y_{n}(x)\right) .
\end{aligned}
$$


Transposing we obtain:

$$
\begin{gathered}
a_{11} \frac{d y_{2}}{d x}+\cdots+a_{1 r} \frac{d y_{r+1}}{d x}=b_{1}\left(x, y_{2}(x), \ldots, y_{n}(x)\right)-a_{1 r+1} \frac{d y_{r+2}}{d x}-\cdots-a_{1 n-1} \frac{d y_{n}}{d x} \\
\vdots \\
a_{r 1} \frac{d y_{2}}{d x}+\cdots+a_{r r} \frac{d y_{r+1}}{d x}=b_{r}\left(x, y_{2}(x), \ldots, y_{n}(x)\right)-a_{r r+1} \frac{d y_{r+2}}{d x}-\cdots-a_{r n-1} \frac{d y_{n}}{d x} .
\end{gathered}
$$

Solving for $d y_{2} / d x, \ldots, d y_{r+1} / d x$ using Cramer's rule,

$\left(\mathrm{E}^{\prime}\right)$

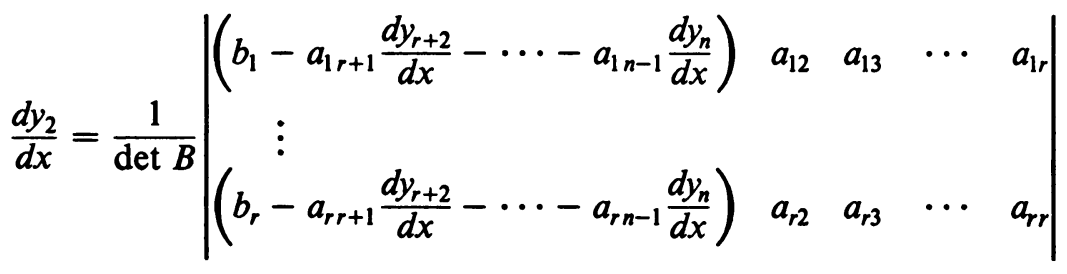

$$
\begin{aligned}
& \frac{d y_{3}}{d x}=\cdots \\
& \frac{d y_{r+1}}{d x}=\cdots \text {. }
\end{aligned}
$$

Let $u_{r+2}(x), \ldots, u_{n}(x)$ be any fixed continuous functions with continuous derivatives such that $u_{r+2}\left(x_{0}\right)=y_{r+2}\left(x_{0}\right), \ldots, u_{n}\left(x_{0}\right)=y_{n}\left(x_{0}\right)$ and such that det $B\left(x, y_{2}, \ldots, y_{r+1}, u_{r+2}, \ldots, u_{n}\right)$ does not vanish on $I_{2}$. Replace $y_{r+2}(x)$ by $u_{r+2}(x), \ldots, y_{n}(x)$ by $u_{n}(x)$ in the right-hand side of the equations of $\left(\mathrm{E}^{\prime}\right)$. The equations of $\left(E^{\prime}\right)$ considered as functions of $x, y_{2}, \ldots, y_{r+1}$ become

$$
\begin{array}{cc}
\frac{d y_{2}}{d x} & =\frac{T_{2}\left(x, y_{2}, \ldots, y_{r+1}\right)}{S\left(x, y_{2}, \ldots, y_{r+1}\right)} \\
\vdots & \vdots \\
\frac{d y_{r+1}}{d x}= & \frac{T_{r+1}\left(x, y_{2}, \ldots, y_{r+1}\right)}{S\left(x, y_{2}, \ldots, y_{r+1}\right)}
\end{array}
$$

Since $S\left(x_{0}, y_{2}\left(x_{0}\right), \ldots, y_{r+1}\left(x_{0}\right)\right) \neq 0$ there is an $I_{2}^{*} \times J_{2}^{*} \times \cdots \times J_{r+1}^{*} \subseteq I_{2} \times J_{2}$ $\times \cdots \times J_{r+1}$ such that $\left\langle x_{0}, y_{2}\left(x_{0}\right), \ldots, y_{r+1}\left(x_{0}\right)\right\rangle \in I_{2}^{*} \times J_{2}^{*} \times \cdots \times J_{r+1}^{*}$ and $S\left(x, y_{2}, \ldots, y_{r+1}\right)$ does not vanish on $I_{2}^{*} \times J_{2}^{*} \times \cdots \times J_{r+1}^{*}$. By the Cauchy-Peano theorem there exists a solution $u_{2}, \ldots, u_{r+1}$ on some $I_{3} \subseteq I_{2}^{*}$ such that $x_{0} \in I_{3}$ and such that $u_{i}\left(x_{0}\right)=y_{i}\left(x_{0}\right)$ for $i=2, \ldots, r+1$. This contradicts the unicity of solution which follows from the fact that $\left\langle x_{0}, y_{2}\left(x_{0}\right), \ldots, y_{n}\left(x_{0}\right)\right\rangle \in \mathrm{Dg}$.

Hence $\operatorname{rank} A=n-1$ on $I_{1}$. Let $I^{\prime} \subseteq I_{1}$ be such that $\operatorname{det} A \neq 0$ for any $x \in I^{\prime}$. We now restrict our attention to $I^{\prime}$. Solving equations (E) we obtain: 
$\left(E^{\prime \prime}\right)$

$$
\begin{aligned}
& \frac{d y_{2}}{d x}=\frac{P_{1}\left(x, y_{2}, \ldots, y_{n}\right)}{Q\left(x, y_{2}, \ldots, y_{n}\right)} \\
& \vdots \\
& \frac{d y_{n}}{d x}=\frac{P_{n-1}\left(x, y_{2}, \ldots, y_{n}\right)}{Q\left(x, y_{2}, \ldots, y_{n}\right)}
\end{aligned}
$$

where $P_{1}, \ldots, P_{n-1}$ are polynomials in $x, y_{2}, \ldots, y_{n}$ and $Q\left(x, y_{2}, \ldots, y_{n}\right)=\operatorname{det} A$.

We now show that there is a nontrivial polynomial $P$ such that

$$
P\left(x, y_{2}, d y_{2} / d x, \ldots, d^{n+1} y_{2} / d x^{n+1}\right)=0 \text { on } I^{\prime} .
$$

Differentiating $d y_{2} / d x=P_{2}\left(x, y_{2}, \ldots, y_{n}\right) / Q\left(x, y_{2}, \ldots, y_{n}\right)$ with respect to $x$ and using $\left(E^{\prime \prime}\right)$ we get

$$
d^{k} y_{2} / d x^{k}=R_{k}\left(x, y_{2}(x), \ldots, y_{n}(x)\right), \quad k=1, \ldots, n+1,
$$

where the $R_{k}$ are rational in $x, y_{2}, \ldots, y_{n}$ and the denominator of each $R_{k}$ is of the form $\left(Q\left(x, y_{2}, \ldots, y_{n}\right)\right)^{r}$ for some natural number $r$. We can assume that each $R_{k} \not \equiv 0$ on $I^{\prime}$ and that $R_{i} \neq R_{j}$ for $i \neq j$-for otherwise we are finished.

Let $v_{2}, \ldots, v_{n}$ be indeterminates. Consider the rational functions

$$
R_{1}\left(x, v_{2}, \ldots, v_{n}\right), \ldots, R_{n+1}\left(x, v_{2}, \ldots, v_{n}\right) .
$$

By simple facts concerning the degree of transcendence of transcendental field extensions there exists a nontrivial polynomial $P$ with coefficients in the reals such that

$$
P\left(R_{1}\left(x, v_{2}, \ldots, v_{n}\right), \ldots, R_{n+1}\left(x, v_{2}, \ldots, v_{n}\right)\right)=0 .
$$

From this it follows that

$$
P\left(R_{1}\left(x, y_{2}(x), \ldots, y_{n}(x)\right), \ldots, R_{n+1}\left(x, y_{2}(x), \ldots, y_{n}(x)\right)\right)=0,
$$

i.e.

$$
P\left(d y_{2} / d x, d^{2} y_{2} / d x^{2}, \ldots, d^{n+1} y_{2} / d x^{n+1}\right)=0 .
$$

Note that $y_{2}$ is the restriction of an analytic function on a subinterval $I^{+}$. This is a well-known fact which holds because $y_{2}$ satisfies an algebraic differential polynomial. We will not go further into the proof of this fact. We merely use it in the proof of Theorem 8 below.

Theorem 3. There exist entire functions which are computable, but are such that the restriction to any closed bounded real interval cannot be generated by an analog computer.

Proof. The proof is an immediate consequence of Theorem 2 and a result of Pólya and/or Hölder. Pólya's result provides us with many examples. Hölder's result shows that $1 / \Gamma(x)$ is also an example. We will discuss both. 
Suppose $f$ is an entire function which satisfies an algebraic differential polynomial on some closed bounded interval $I$. Let $f^{*}$ be the (entire) function of a complex variable such that $f^{*}(x)=f(x)$ on the real line. Then by the identity theorem for analytic functions $f^{*}(z)$ satisfies an algebraic differential equation on the whole plane. Thus to prove Theorem 3 we must exhibit entire computable functions $f^{*}(z)$ which do not satisfy any algebraic differential polynomial. For by Theorem 2, they will not be analog generable on any $I$.

The theorem of Pólya(13) referred to above may be stated as follows: if the coefficients of an entire transcendental function $w=b_{0}+b_{1} z+\cdots+b_{n} z^{n}$ $+\cdots$ are rational numbers and if the function satisfies an algebraic differential polynomial then $\lim \sup _{n \rightarrow \infty}\left(\log \left|b_{n}\right| / n(\log n)^{2}\right)$ is finite.

As indicated above Pólya's theorem allows us to construct many examples. We give one. Let

$$
f(x)=\sum_{r=1}^{\infty} \frac{x^{r}}{r^{\left(r^{3}\right)}} ; \quad f^{*}(z)=\sum_{r=1}^{\infty} \frac{z^{r}}{r^{\left(r^{3}\right)}} \text {. }
$$

It is easy to see that $f^{*}(z)$ is entire. Furthermore

$$
\varlimsup_{n \rightarrow \infty} \frac{\log \left|1 / n^{\left(n^{3}\right)}\right|}{n(\log n)^{2}}
$$

is not finite. Hence $f$ cannot satisfy an algebraic differential polynomial. Furthermore it is trivial to show that $f$ is computable. Define $p_{n}$ by

$$
p_{n}(x)=\sum_{t=1}^{n+1} \frac{x^{t}}{t^{\left(t^{3}\right)}}
$$

Let $g(N, M)=(2(N+1))^{M}$. Then if $|x| \leq N$ (where $N$ is positive), $M \geq 1$, and $n \geq g(N, M)$,

$$
\begin{aligned}
\left|f(x)-p_{n}(x)\right| & \leq \sum_{n+2}^{\infty} \frac{|x|^{t}}{t^{\left(t^{3}\right)}} \leq \sum_{n+2}^{\infty} \frac{(N+1)^{t}}{(2(N+1))^{M t^{3}}} \\
& \leq \sum_{t=n+2}^{\infty} \frac{1}{2^{M t^{3}}} \leq \sum_{t=n+2}^{\infty} \frac{1}{2^{M t}}<\frac{1}{2^{M}} .
\end{aligned}
$$

Note that the functions $q, r, s, d$ and $g$ of Definitions 4 and 5 which are used in showing that $f$ is computable are all elementary. Hence $f$ is not merely computable but elementary recursive.

To show that $1 / \Gamma(x)$ is also an example we recall the following. $\Gamma(x)$ is analytic except at $x=0,-1,-2, \ldots$ where it has simple poles. $\Gamma(x)$ does not satisfy an algebraic differential polynomial (Hölder [6]). The function $1 / \Gamma(x)$ is entire. Thus $1 / \Gamma(x)$ cannot satisfy an algebraic differential polynomial on any closed bounded interval $I$. Hence the only task which remains is to show that $1 / \Gamma(x)$ is

(13) We have used Pólya [14], but Pólya [15] may also be used instead. 
computable. By definition

$$
\frac{1}{\Gamma(x)}=x e^{\gamma x} \prod_{n=1}^{\infty}\left\{\left(1+\frac{x}{n}\right) e^{-x / n}\right\}
$$

where $\gamma=\lim _{m \rightarrow \infty}\{1+1 / 2+\cdots+1 / m-\log m\}$. The proof that $1 / \Gamma(x)$ is computable is very long. The author has written up the details and is willing to supply them on request.

We note that $\Gamma(x)$ is not analog generable on any interval on which it is defined.

Theorem 4. Suppose $y(x)$ satisfies an algebraic differential polynomial on I. Then there is an $I^{\prime} \subseteq I$ such that on $I^{\prime}, y(x)$ can be generated by a G.P.A.C.

Proof. Since the proof is detailed, a brief outline may be useful. We take a certain algebraic differential polynomial-say of order $n+1$-and we show that for some $J_{0} \subseteq I, y(x)$ is the unique solution of this polynomial satisfying a set of initial conditions $y(a), \ldots, y^{(n)}(a)$. We then proceed to relabel the derivatives. Thus $y(x)$ becomes $y_{2}(x), y^{\prime}(x)$ becomes $y_{3}(x), \ldots$, etc. Hence, in effect, we are rewriting the differential equation in terms of $x, y_{2}, \ldots, y_{n}$ and adding the defining equations $d y_{i} / d x=y_{i+1}$. The remaining task is two-fold. First we must convert this set of equations into a set of equations of the form required by Definition 10. Second we must show that the set of initial conditions guaranteed by Definition 10 has a "domain of generation".

We now proceed with the proof. Assume without loss of generality that $P$ is an algebraic differential polynomial of lowest order-say of order $n$-and of lowest degree in that order such that $P\left(x, y, y^{\prime}, \ldots, y^{(n)}\right)=0$ on $I$. Differentiating $P$ formally we obtain

$$
R y^{(n+1)}-Q=0
$$

where $Q\left(x, y, y^{\prime}, \ldots, y^{(n)}\right)$ and $R\left(x, y, y^{\prime}, \ldots, y^{(n)}\right)$ are algebraic differential polynomials of order at most $n$. Furthermore it is easily seen that either $R$ is of order lower than $n$ or, if $R$ is of order $n$, then it is of lower degree than $P$ in $y^{(n)}$. In either case we see that $R\left(x, y, y^{\prime}, \ldots, y^{(n)}\right) \not \equiv 0$ on $I$. Let $a, y(a), y^{\prime}(a), \ldots, y^{(n)}(a)$ 一where $a \in I$-be such that $R\left(a, y(a), \ldots, y^{(n)}(a)\right) \neq 0$. Then there is a $J_{0} \times J_{1}$ $\times \cdots \times J_{n+1}$ such that:

(i) $J_{0} \subseteq I$.

(ii) $\left\langle a, y(a), \ldots, y^{(n)}(a)\right\rangle \in J_{0} \times J_{1} \times \cdots \times J_{n+1}$.

(iii) If $\left\langle c_{0}, c_{1}, c_{2}, \ldots, c_{n+1}\right\rangle \in J_{0} \times J_{1} \times \cdots \times J_{n+1}$ then $R\left(c_{0}, c_{1}, \ldots, c_{n+1}\right) \neq 0$.

(iv) $x \in J_{0} \Rightarrow\left\langle x, y(x), \ldots, y^{(n)}(x)\right\rangle \in J_{0} \times \cdots \times J_{n+1}$.

Hence on $J_{0}, y(x)$ is the unique solution possessing initial conditions $y(a), y^{\prime}(a)$, $\ldots, y^{(n-1)}(a), y^{(n)}(a)$ at $x=a$ which satisfies $(*)$ on $J_{0}$.

Note (*) satisfies the Lipschitz condition because of the choice of $J_{0} \times \cdots$ $\times J_{n+1}$. Furthermore $y^{(n+1)}(x)$ exists on $J_{0}$ and satisfies (*). 
We wish to prove that, on $J_{0}, y(x)$ can be generated by a G.P.A.C. Our first task is to find a set of equations of the form required by Definition 10. Solving for $y^{(n+1)}$ in (*) we obtain

$$
y^{(n+1)}=\frac{Q\left(x, y, y^{\prime}, \ldots, y^{(n)}\right)}{R\left(x, y, y^{\prime}, \ldots, y^{(n)}\right)} .
$$

Now we introduce variables as follows: let $y_{1} \equiv x, y_{2} \equiv y, y_{3} \equiv y^{\prime}, \ldots, y_{n+2}$ $\equiv y^{(n)}, y_{n+3} \equiv y^{(n+1)}$. Then equation (A) becomes

$$
\begin{aligned}
d y_{k} / d y_{1} & =y_{k+1}, \quad k=2, \ldots, n+2, \\
y_{n+3} & =\frac{Q\left(y_{1}, y_{2}, \ldots, y_{n+2}\right)}{R\left(y_{1}, y_{2}, \ldots, y_{n+2}\right)} .
\end{aligned}
$$

So the problem remains to show that the last equation of this set can be replaced by a set of equations of the form required by Definition 10 . Consider first the polynomial $Q$. Each term of $Q$ which is not a constant is of the form $b v_{1} \cdots \cdots v_{r}$ where each $v_{i}$ is one of the $y_{j}$ and $b$ is a real number. Let

$$
\frac{d y_{n+4}}{d y_{1}}=b v_{1} \frac{d v_{2}}{d y_{1}}+b v_{2} \frac{d v_{1}}{d y_{1}}
$$

Set the initial conditions of $y_{n+4}$ so that $y_{n+4}(a)=b v_{1}(a) \cdot v_{2}(a)$. Thus $y_{n+4}(x)$ $=b v_{1}(x) v_{2}(x)$. Then let

$$
\frac{d y_{n+5}}{d y_{1}}=v_{3} \frac{d y_{n+4}}{d y_{1}}+y_{n+4} \frac{d v_{3}}{d y_{1}}
$$

and set $y_{n+5}(a)=v_{3}(a) \cdot y_{n+4}(a)$. Then $y_{n+5}=b v_{1} v_{2} v_{3}$. If we continue this procedure we finally obtain $y_{n+r+2}=b v_{1} \cdots \cdots v_{r}$. Let the $y$ 's corresponding to the nonconstant terms of $Q$ be $w_{1}, \ldots, w_{s}$; let those corresponding to the nonconstant terms of $R$ be $w_{1}^{*}, \ldots, w_{t}^{*}$. Then

$$
y_{n+3}=\left(\sum_{i=1}^{s} w_{i}+c\right) /\left(\sum_{i=1}^{t} w_{i}^{*}+c^{*}\right)
$$

where $c$ and $c^{*}$ are real constants which may be zero. We must now reduce (B) to a set of equations of the form required by Definition 10 . Suppose the last $y, w_{t}^{*}$, was $y_{p-1}$. Let(14)

(14) A simple modification of the technique used in equations (C) will allow us to conclude: if $y$ can be generated by a G.P.A.C. on $I$ and if $y(x) \neq 0$ for $x \in I, 1 / y(x)$ can be generated by a G.P.A.C on $I$. 
(C)

$$
\left\{\begin{aligned}
\frac{d y_{p}}{d y_{1}} & =-y_{p+1} d\left(\sum_{i=1}^{t} w_{i}^{*}+c^{*}\right) / d y_{1}, \\
\frac{d y_{p+1}}{d y_{1}} & =2 y_{p} \frac{d y_{p}}{d y_{1}} .
\end{aligned}\right.
$$

Set the initial conditions of $y_{p}$ and $y_{p+1}$ by

$$
y_{p}(a)=\left(\sum_{i=1}^{t} w_{i}^{*}(a)+c^{*}\right)^{-1}, \quad y_{p+1}(a)=y_{p}^{2}(a) .
$$

Thus $y_{p}(x)=\left(\sum_{i=1}^{t} w_{i}^{*}(x)+c^{*}\right)^{-1}$. We replace (B) by (C) together with the equation

$$
\frac{d y_{n+3}}{d y_{1}}=y_{p} d\left(\sum_{i=1}^{s} w_{i}+c\right) / d y_{1}+\left(\sum_{i=1}^{s} w_{i}+c\right) \frac{d y_{p}}{d y_{1}}
$$

where $y_{n+3}(a)=\left(\sum_{i=1}^{s} w_{i}(a)+c\right) \cdot y_{p}(a)$.

We now consider the set of equations we have created-viz.

$$
\begin{aligned}
\frac{d y_{2}}{d x} & =y_{3} \\
\vdots & \\
\frac{d y_{n+2}}{d x} & =y_{n+3} \\
\frac{d y_{n+4}}{d x} & =b v_{1} \frac{d v_{2}}{d x}+b v_{2} \frac{d v_{1}}{d x} \\
\vdots & \\
\frac{d y_{p-1}}{d x} & =y_{p-2} \frac{d v_{m}}{d x}+v_{m} \frac{d y_{p-2}}{d x} \text { where } v_{m} \in\left\{y_{2}, \ldots, y_{n+2}\right\} \\
\frac{d y_{p}}{d x} & =-y_{p+1} d\left(\sum_{i=1}^{t} w_{i}^{*}+c\right) / d y_{1} \\
\frac{d y_{p+1}}{d y_{1}} & =2 y_{p} \frac{d y_{p}}{d x} \\
\frac{d y_{n+3}}{d x} & =y_{p} d\left(\sum w_{i}+c\right) / d y_{1}+\left(\sum_{i=1}^{s} w_{i}+c\right) \frac{d y_{p}}{d y_{1}} .
\end{aligned}
$$

(D)

Note that we have placed the equation whose left member is $d y_{n+3} / d x$ at the end. All other equations are in the normal order.

By construction for some $I^{\prime} \subseteq J_{0}$ there is a unique solution $\left\{y_{2}, \ldots, y_{n+2}\right.$, $\left.y_{n+4}, \ldots, y_{p}, y_{p+1}, y_{n+3}\right\}$ satisfying the initial conditions $y_{2}(a)=y(a), y_{3}(a)=y^{\prime}(a)$, etc. and such that $y_{2}(x)=y(x)$ for $x \in I^{\prime}$.

We must now produce a domain of generation for $\left\langle a, y_{2}(a), y_{3}(a), \ldots, y_{n+2}(a)\right.$, $\left.y_{n+4}(a), \ldots, y_{p}(a), y_{p+1}(a), y_{n+3}(a)\right\rangle$. To do this we rewrite the set of equations as: 


$$
\vec{A}\left(x, y_{2}, \ldots, y_{n+2}, y_{n+4}, \ldots, y_{p}, y_{p+1}, y_{n+3}\right)\left[\begin{array}{c}
\frac{d y_{2}}{d x} \\
\vdots \\
\frac{d y_{n+2}}{d x} \\
\frac{d y_{n+4}}{d x} \\
\vdots \\
\frac{d y_{p}}{d x} \\
\frac{d y_{p+1}}{d x} \\
\frac{d y_{n+3}}{d x}
\end{array}\right]=\vec{b}\left(x, y_{2}, \ldots, y_{p+1}, y_{n+3}\right) \text {. }
$$

If we look at the matrix $\vec{A}$ we find it is of the form

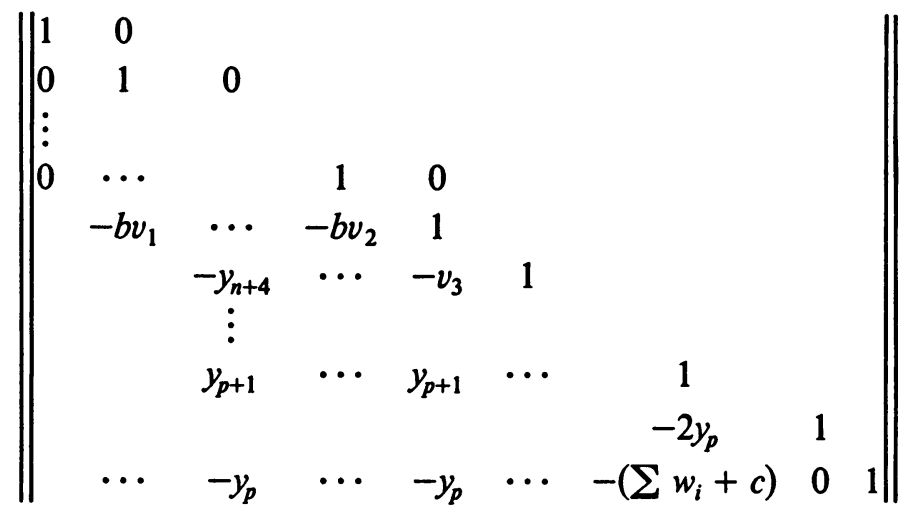

Hence the determinant of this matrix is 1 . Therefore the set of equations we are considering is equivalent to

$$
\begin{aligned}
d y_{2} / d x & =b_{2}^{*}\left(x, y_{2}, \ldots, y_{p}, y_{p+1}, y_{n+3}\right) \\
\vdots & \\
d y_{n+2} / d x & =b_{n+2}^{*}\left(x, y_{2}, \ldots, y_{p}, y_{p+1}, y_{n+3}\right) \\
d y_{n+4} / d x & =b_{n+4}^{*}\left(x, y_{2}, \ldots, y_{p}, y_{p+1}, y_{n+3}\right) \\
\vdots & \\
d y_{p+1} / d x & =b_{p+1}^{*}\left(x, y_{2}, \ldots, y_{p}, y_{p+1}, y_{n+3}\right) \\
d y_{n+3} / d x & =b_{n+3}^{*}\left(x, y_{2}, \ldots, y_{p}, y_{p+1}, y_{n+3}\right)
\end{aligned}
$$

where the $b_{i}^{*}$ 's are polynomials in the $y_{j}$ 's. Thus the Lipschitz condition is satisfied. Hence given $\mathbf{c}=\left\langle c, c_{2}, \ldots, c_{n+2}, c_{n+4}, \ldots, c_{p}, c_{p+1}, c_{n+3}\right\rangle$, there is a $J_{1}^{(c)} \times \ldots$ $\times J_{n+2}^{(c)} \times J_{n+4}^{(c)} \times \cdots \times J_{p+1}^{(c)} \times J_{n+3}^{(c)}$ containing $\mathrm{c}$ such that there is a unique solution $\left\{y_{2}^{\dagger}, \ldots, y_{n+2}^{\dagger}, y_{n+4}^{\dagger}, \ldots, y_{p+1}^{\dagger}, y_{n+3}^{\dagger}\right\}$ of $\left(\mathrm{D}^{\prime}\right)$ on $J_{1}^{(c)} \times \cdots \times J_{n+3}^{(c)}$ satisfying $y_{i}^{\dagger}(c)=c_{i}$. 
Thus associated with every set of initial conditions c, there exists a domain of generation with respect to $\left(D^{\prime}\right)$ and hence with respect to (D) itself.

Trivially $e^{x}, \log x$, and all the trigonometric functions can be generated by a G.P.A.C. on every $I$ for which they are defined. (Note that both $\sin x$ and $\cos x$ are solutions of the same differential equation $d^{2} y / d x^{2}+y=0$. However $\sin x$ is the unique solution satisfying $y(a)=\sin a, y^{\prime}(a)=\cos a$ whereas $\cos x$ is the unique solution satisfying $y(a)=\cos a, y^{\prime}(a)=-\sin a$. Note further that $1 / \sin x$ and $1 / \cos x$ can be shown to be generable by a technique similar to that used in equations (C) of the preceding theorem.)(14) But we need not confine our attention to elementary functions. The above considerations show that special functions which arise as solutions of differential equations can also be generated by G.P.A.C.--e.g. Bessel functions, etc. The reader is invited to consult the literature on special functions for numerous examples.

4. Essential computability of analog generable functions. In this section we show that, roughly, analog generable functions are "essentially computable". The results are given in Theorems 7 and 8 below. Theorem 7 is concerned with entire functions; Theorem 8 deals with a more extensive class of analog generable functions.

In order to obtain our results we concern ourselves with power series which satisfy an algebraic differential polynomial. This approach is very natural. For the characterization of analog generable functions in terms of algebraic differential polynomials-which was given in the last section-leads naturally to a study of solutions of algebraic differential polynomials. Since functions satisfying algebraic differential polynomials are locally analytic-see remark following Theorem 2-we can deal with power series. This explains the motivation behind Theorems 5 and 6 below. Indeed the main results of this section are consequences of Theorems 5 and 6 .

The following terminology will be useful. Let $f(x)=\sum_{i=0}^{\infty} b_{i}(x-c)^{i}$ be a power series ( $c$ and $b_{i}$ are assumed to be real). By the formal extension of $f$ we mean the function $f^{*}$ defined by $f^{*}(z)=\sum_{i=0}^{\infty} b_{i}(z-c)^{i}$.

Theorem 5.(15) Suppose $f^{*}(z)$ satisfies an algebraic differential polynomial. Then the sequence of coefficients $\left\{b_{i}\right\}$ is essentially computable in a finite number of the $b_{i}$ 's and $c$.

Proof. The theorem is trivial if $f^{*}(z)$ is a polynomial. So suppose $f^{*}(z)$ is not a polynomial. By assumption there is a polynomial $\boldsymbol{P}$ such that

$$
P\left(w^{(n)}, w^{(n-1)}, \ldots, w, z\right)=0 .
$$

We assume that (1) is an algebraic differential equation of as low an order as

(15) In this proof we adapt to our needs some of the techniques used by Hurwitz in the proof of a very different theorem [7] involving power series with rational coefficients. We present enough details to indicate that the proof is effective in the recursion-theoretic sense. 
possible, and of as low a degree as possible in that order which is satisfied by $w=f^{*}(z)$. Now consider $P_{n}$ defined by

$$
\partial P / \partial w^{(n)}=P_{n} .
$$

If one substitutes $f^{*}(z)$ in $P_{n}$, there is a nonnegative integer $r$ and a number $d \neq 0$ so that

$$
P_{n}=d(z-c)^{r}+\cdots .
$$

( $P_{n}$ cannot vanish identically because of the choice of $P$.)

In the remainder of the proof we use the notation $Q_{t, u}, Q_{t}$ and $R_{t, u}$ to refer to certain polynomials in $w^{(t)}, w^{(t-1)}, \ldots, w, z$ with coefficients which are rational in the coefficients of $P$. (Note that the highest derivative which appears is at most of order $t$.)

We first show that, for $s=n+2(r+1)$ where $r$ is as in (3), there exist $Q_{n+1}, \ldots, Q_{n+r^{\prime}}, Q_{s-r-1}$ such that

$$
w^{(s)} P_{n}+w^{(s-1)} Q_{n+1}+\cdots+w^{(s-r)} Q_{n+r}+Q_{s-r-1}=0 .
$$

This is proved by showing that for every $m$, there exist $Q_{n+1, m}, \ldots, Q_{n+m-1, m}$, $Q_{n+m, m}$ such that

$$
w^{(n+2 m)} P_{n}+w^{(n+2 m-1)} Q_{n+1, m}+\cdots+w^{(n+m+1)} Q_{n+m-1, m}+Q_{n+m, m}=0 .
$$

The proof of $(5)_{m}$ is by induction on $m$. For $m=1$ the equation holds trivially. In order to prove (5) $)_{m+1}$ from (5) $m$ we differentiate $(5)_{m}$ twice and rearrange the terms carefully. The tedious details are left to the reader. (4) follows immediately from $(5)_{r+1}$. (Here we abbreviate $Q_{n+1, r+1}$ by $Q_{n+1}$, etc.)

Next, differentiating (4) $t$ times we obtain (6) below. (The proof of (6) proceeds by a straightforward induction on $t$, using the fact that $\left(\begin{array}{c}t \\ m-1\end{array}\right)+\left(\begin{array}{c}t \\ m-2\end{array}\right)=\left(\begin{array}{c}t+1 \\ m-1\end{array}\right)$. $)$

$$
\begin{aligned}
w^{(s+t)} P_{n} & +w^{(s+t-1)}\left(Q_{n+1}+t P_{n}^{\prime}\right)+w^{(s+t-2)}\left(Q_{n+2}+t Q_{n+1}^{\prime}+\left(\begin{array}{l}
t \\
2
\end{array}\right) P_{n}^{\prime \prime}\right)+\cdots \\
& +w^{(s+t-r)}\left(Q_{n+r}+t Q_{n+r-1}^{\prime}+\cdots+\left(\begin{array}{l}
t \\
r
\end{array}\right) P_{n}^{(r)}\right)+R_{s+t-r-1, t}=0,
\end{aligned}
$$

where the order of the highest derivative occurring in $R$ is at most of order $s+t-(r+1)$. Here $P_{n}$ and the $Q$ 's are as in (4) and for $t>0, R_{s+t-r-1, t}$ is defined by

$$
\begin{aligned}
R_{s+t-(r+1), t}= & w^{(s+t-(r+1))}\left[Q_{n+r}^{\prime}+(t-1) Q_{n+r-1}^{\prime \prime}+\cdots+\left(\begin{array}{c}
t-1 \\
r
\end{array}\right) P_{n}^{(r+1)}\right] \\
& +R_{s+(t-1)-(r+1), t-1}^{\prime} .
\end{aligned}
$$

Thus $R_{s+t-(r+1), t}$ can be determined effectively from $R_{s+(t-1)-(r+1), t-1}$ and the $Q$ 's and $P_{n}$ of (4). Now substitute $f^{*}(z)$ for $w$ in (6) and set $z=c$. Then (6) becomes 


$$
\begin{gathered}
w_{c}^{(s+t)} p_{n}+w_{c}^{(s+t-1)}\left(d_{n+1}+t p_{n}^{\prime}\right)+w_{c}^{(s+t-2)}\left(d_{n+2}+t d_{n+1}^{\prime}+\left(\begin{array}{l}
t \\
2
\end{array}\right) p_{n}^{\prime \prime}\right)+\cdots \\
+w_{c}^{(s+t-r)}\left(d_{n+r}+t d_{n+r-1}^{\prime}+\cdots+\left(\begin{array}{l}
t \\
r
\end{array}\right) p_{n}^{(r)}\right)+\rho_{s+t-r-1, t, c}=0
\end{gathered}
$$

where $p_{n}^{(r)}$ is an abbreviation for $p_{n}$ with a string of $r$ primes. Here the $d$ 's and $p_{n}$ 's-with and without primes-are real numbers. Not all the parenthesized polynomials in $t$ vanish identically. In particular $\left(d_{n+r}+t d_{n+r-1}^{\prime}+\cdots+\left(\begin{array}{l}t \\ r\end{array}\right) p_{n}^{(r)}\right)$ is not the zero polynomial since $p_{n}^{(r)}$, which is a multiple of the $d$ of (3), is nonzero. Now let $v \leq r$ be the smallest integer for which

$$
d_{n+v}+d_{n+v-1}^{\prime} t+\cdots+\left(\begin{array}{l}
t \\
v
\end{array}\right) p_{n}^{(v)}
$$

is not identically zero. Then, transposing (7) we have

$$
w_{c}^{(s+t-v)}\left(d_{n+v}+\cdots+\left(\begin{array}{l}
t \\
v
\end{array}\right) p_{n}^{(v)}\right)=S_{t}\left(w_{c}^{(s+t-v-1)}, w_{c}^{(s+t-v-2)}, \ldots, w_{c}^{\prime}, w_{c}\right)
$$

where $S_{t}$ is a polynomial. Let $m=s+t-v$. Then (9) becomes

$$
w_{c}^{(m)}\left(g_{0}+g_{1} m+\cdots+g_{v} m^{v}\right)=S_{m}^{*}\left(w_{c}^{(m-1)}, w_{c}^{(m-2)}, \ldots, w_{c}^{\prime}, w_{c}\right)
$$

where the $g$ 's are real numbers and $S_{m}^{*}$ is the result of replacing $t$ by $m-s+v$ in $S_{t}$. Now let $m_{0}$ be chosen so large that for all $m>m_{0}, g_{0}+g_{1} m+\cdots$ $+g_{v} m^{v} \neq 0$. Then

$$
w_{c}^{(m)}=\frac{S_{m}^{*}\left(w_{c}^{(m-1)}, w_{c}^{(m-2)}, \ldots, w_{c}^{\prime}, w_{c}\right)}{g_{0}+g_{1} m+\cdots+g_{v} m^{v}}
$$

for all $m>m_{0}$. Now $b_{m}=w_{c}^{(m)} / m$ !. Hence for $m>m_{0}$,

$$
b_{m}=\frac{S_{m}^{*}\left(w_{c}^{(m-1)}, w_{c}^{(m-2)}, \ldots, w_{c}^{\prime}, w_{c}\right)}{m !\left(g_{0}+g_{1} m+\cdots+g_{v} m^{v}\right)} .
$$

Thus we see that, for $m>m_{0}, b_{m}$ can be obtained effectively from $c$, the coefficients of $P$, and from $b_{k}$ for $k \leq m_{0}$. Hence the sequence $\left\{b_{i}\right\}$ is essentially computable in $b_{0}, b_{1}, \ldots, b_{m_{0}}, c$ and the coefficients of $P$.

We now remove the dependence on the coefficients of $P$. This is easy to do. For if $\sum_{i=0}^{\infty} b_{i}(x-c)^{i}$ satisfies an algebraic differential polynomial then it satisfies an algebraic differential polynomial in which the coefficients can be obtained from a finite number of the $b_{i}$ and $c$ by rational operations. If we work with this polynomial we can conclude that the sequence $\left\{b_{i}\right\}$ is computable in a finite number of the $b_{i}$ and $c$. Thus there is an $m_{0}$ such that for all $m>m_{0}, b_{m}$ can be obtained effectively by rational operations from $b_{0}, \ldots, b_{m_{0}}, c$.

Theorem 6. Let $f(x)=\sum_{i=0}^{\infty} b_{i}(x-c)^{i}$ be a formal power series where $b_{i}$ and $c$ are real. Let $f^{*}$ be its formal extension. Suppose $R^{*}$, the radius of convergence of $f^{*}$ 
about $z=c$, is positive. Let $\rho$ be so chosen that $0<\rho<R^{*}$. Then the sequence of partial sums of $f$ converges recursively to $f$ on $[c-\rho, c+\rho]$.

Proof. (This proof shows that uniform convergence on $[c-\rho, c+\rho]$ is actually recursive convergence.) Choose a $\rho_{0}$ such that $\rho<\rho_{0}<R^{*}$. It is convenient to let $\rho_{0}=\rho+1 / 2^{k_{0}}$ for some integer $k_{0} \geq 1$. There exists a $p_{0} \geq 1$ such that for all $p>p_{0},\left|b_{p}\right|<1 / \rho_{0}^{p}$. For suppose the contrary. Then for infinitely many $p$, $\left|b_{p}\right| \geq 1 / \rho_{0}^{p}$ and hence $\sum\left|b_{p}\right| \rho_{0}^{p}$ would diverge. Let $M$ be a fixed positive integer greater than $\rho$. Define the recursive function $h$ by

$$
h(t)=2^{t+p_{0}} \cdot 2^{2 k_{0}} \cdot(M+1)^{2} .
$$

Suppose $p \geq h(t)$. Then on $|x-c| \leq \rho$ we have

$$
\left|f(x)-\sum_{i=0}^{p} b_{i}(x-c)^{i}\right|<\frac{1}{2^{i}} \text {. }
$$

Theorem 7. Suppose $f(x)=\sum_{i=0}^{\infty} b_{i} x^{i}$ is entire. Suppose further that for some I, $f \uparrow I$ can be generated by a G.P.A.C. Then for every $J, f \uparrow J$ is essentially computable in the following sense: there exist a finite number of $b_{i}$ such that for every $J, f \uparrow J$ is essentially computable in these $b_{i}$.

Proof. $f \uparrow I$ can be generated by a G.P.A.C. Then by Theorem 2, for some $I_{1} \subseteq I, f$ satisfies an algebraic differential polynomial on $I_{1}$. Since $f$ is entire, $f$ satisfies an algebraic differential polynomial on the whole line. By Theorem $5,\left\{b_{i}\right\}$ is essentially computable in a finite number of $b_{i}$. By Theorem $6, f(x)$ is recursively convergent on every interval $a \leq x \leq b$. Thus $f \uparrow J$ is essentially computable in these $b_{i}$.

In the next theorem the functions need not be entire and $c$ may be chosen to be rational.

Theorem 8. Suppose for some I, $f \uparrow I$ can be generated by a G.P.A.C. Then there is a $J \subseteq I$ such that $f \uparrow J$ is essentially computable in the following sense. $f \uparrow J$ is expressible as $\sum_{i=0}^{\infty} b_{i}(x-c)^{i}$, the restriction of a function of a complex variable which is analytic in a region including $J$, and $f \uparrow J$ is essentially computable in a finite number of the $b_{i}$.

Proof. $f \uparrow I$ can be generated by a G.P.A.C. Hence for some $I^{\dagger} \subseteq I, f$ satisfies an algebraic differential equation on $I^{\dagger}$. By the remark following Theorem 2 we may assume that $f$ is the restriction of a function of a complex variable which is analytic in a region including $I^{\dagger}$. Now choose $c$, a rational number in the interior of $I^{\dagger}$, and expand $f$ in a power series about $c$. Thus for some $R>0$, $f(x)=\sum_{i=0}^{\infty} b_{i}(x-c)^{i}$ is the restriction of an analytic function for $|x-c|<R$. (Note that the $b_{i}$ are all real since $b_{i}=f^{(i)}(c) / i$ !.) Now let $0<\rho<R$. By Theorem $5,\left\{b_{i}\right\}$ is essentially computable in a finite number of the $b_{i}$ 's. By Theorem $6, f(x)$ is recursively convergent on $[c-\rho, c+\rho]$. Hence $f$ is essentially 
computable in a finite number of the $b_{i}$ 's on $[c-\rho, c+\rho]$.

Remark. If, in the proof of Theorems 7 or 8 , each $b_{i}$ in the power series expansion of $f$ is a computable real number, then we may strengthen the conclusion to read: $f \uparrow J$ is computable.

In general, we cannot expect to strengthen either Theorem 7 or 8 to conclude that $f \uparrow J$ is computable. One reason is that by definition the function $C_{k}$ defined by $C_{k}(x)=k$ for a given real $k$ can be generated by a G.P.A.C.

As the reader can easily see, the theorems of this section can be used to obtain results in recursive analysis. The result for entire functions was explained at length in the introduction-see the survey of results of $\$ 4$. It can be extended to cover functions which are not necessarily entire by a trivial modification. We leave the details to the reader.

Areas for further research. The reader who has journeyed with us this far is undoubtedly aware of the tremendous number of open problems which arise quite naturally from the considerations of this paper. Indeed this author has accumulated a list which is several pages long. The most obvious problems may be classified into three main areas-not necessarily disjoint.

Area 1. A study of the effect of adding to (or replacing) the black boxes of $\$ 2$ by other black boxes.

Area 2. A more detailed study of the relation between the approach via analog computers-perhaps as extended in Area 1-and the usual results and methods of recursion theory. (Here, the author has some results concerned with "relative analog generability".)

Area 3. A more detailed study of foundations along the lines discussed in the introduction.

These are only a few of the avenues for further research. It is hoped that the reader will try his hand at some of them.

In conclusion, the author would like to thank her colleagues, Professors J. Eagon, J. Nitsche and Y. Sibuya for valuable conversations. Thanks also to Professor Shepherdson for his hospitality at the University of Bristol, where some of this work was carried out.

\section{BIBLIOGRAPHY}

1. T. M. Apostol, Mathematical analysis: A modern approach to advanced calculus, Addison-Wesley, Reading, Mass., 1957. MR 19, 398.

2. V. Bush, The differential analyzer, a new machine for solving differential equations, J. Franklin Inst. 212 (1931), 447-488.

3. A. Church, An unsolvable problem in elementary number theory, Amer. J. Math. 58 (1936), 345-363.

4. J. Crank, The differential analyzer, Longmans, Green, London, 1947. MR 10,70.

5. A. Grzegorczyk, On the definitions of computable real continuous functions, Fund. Math. 44 (1957), 61-71. MR 19, 723.

6. O. Hölder, Ueber die Eigenschaft der Gamma funktion keiner algebraischen Differentialgleichung zu genügen, Math. Ann. 28 (1887), 1-13. 
7. A. Hurwitz, Sur le développement des fonctions satisfaisant à une équation différentielle algébrique, Ann. Ecole Norm. (3) 6 (1889), 327-332.

8. A. Jackson, Analog computation, McGraw-Hill, New York, 1960.

9. C. L. Johnson, Analog computer techniques, McGraw-Hill, New York, 1963. MR 22 \# 12759.

10. S. C. Kleene, Introduction to metamathematics, Van Nostrand, Princeton, N.J., 1952. MR 14, 525.

11. G. A. Korn and T. M. Korn, Electronic analog and hybrid computers, McGraw-Hill, New York, 1964.

12. D. Lacombe, Extension de la notion de fonctions récursive aux fonctions d'une ou plusieurs variables réelles. I,II,III, C.R. Acad. Sci. Paris 240 (1955), 2478-2480; ibid. 241 (1955), 13-14, 151-153. MR 17, 225.

13. A. A. Markov, The theory of algorithms, Trudy Mat. Inst. Steklov. 38 (1951), 176-189; English transl., Amer. Math. Soc. Transl. (2) 15 (1960), 1-14. MR 13, 811; MR 22 \#5572.

14. G. Pólya, Uber das Anwachsen von ganzen Funktionen die einer Differentialgleichung genügen, Viert. Naturforsch. Ges. Zürich 61 (1916), 531-545.

15.—, Zur Untersuchung der Grössenordnung ganzer Funktionen, die einer Differentialgleichung genügen, Acta Math. 42 (1920), 309-316.

16. H. G. Rice, Recursive real numbers, Proc. Amer. Math. Soc. 5 (1954), 784-791. MR 16, 104.

17. C. E. Shannon, Mathematical theory of the differential analyzer, J. Math. Phys. Mass. Inst. Tech. 20 (1941), 337-354. MR 3, 279.

18. W. Soroka, Analog methods in computation and simulation, McGraw-Hill, New York, 1954. MR 16, 526.

19. E. Specker, Nicht konstrucktiv beweisbare Sätze der Analysis, J. Symbolic Logic 14 (1949), 145-158. MR 11, 151.

20. W. Thomson (Lord Kelvin), On an instrument for calculating the integral of the product of two given functions, Proc. Roy. Soc. London 24 (1876), 266-268. See also pp. 269-271 and pp. 271-275.

21. R. Tomovič and W. J. Karplus, High speed analog computers, Wiley, New York, 1962.

22. A. Turing, On computable numbers with an application to the Entscheindungsproblem, Proc. London Math. Soc. (2) 42 (1937), 230-265; ibid. (2) 43 (1938), 544-546.

School of Mathematics, University of Minnesota, MinNeapolis, MinNesota 55455 